\title{
Experimental observation of temporal dispersion gratings in fiber optics
}

\author{
Christophe Finot ${ }^{1, *}$ AND HeRVÉ Rigneault ${ }^{2}$ \\ ${ }^{1}$ Laboratoire Interdisciplinaire Carnot de Bourgogne, UMR 6303 CNRS-Université de Bourgogne-Franche-Comté, 9 avenue Alain Savary, BP \\ 47870, 21078 Dijon Cedex, France \\ ${ }^{2}$ Aix Marseille Université, CNRS, Centrale Marseille, Institut Fresnel UMR 7249, 13397 Marseille, France
}

*Corresponding author: christophe.finot@u-bourgogne.fr

Received XX Month XXXX; revised XX Month, XXXX; accepted XX Month XXXX; posted XX Month XXXX (Doc. ID XXXXX); published XX Month XXXX

We experimentally demonstrate a temporal analog to the diffraction optical grating. Using amplitude and phase temporal periodic modulations, we show that the accumulation of dispersion in fiber optics induces the development of temporally well-separated sidebands similar to the spatial orders of diffraction that are commonly observed in optical grating.

\section{Introduction}

Diffraction is among the key effects of physical optics with applications in a broad range of technological domains spanning from imaging to spectroscopy, material sciences or sensors. Wave propagation through a periodic pattern is the unavoidable example taught in any physical optics courses $[1,2]$ and is usually illustrated by the transmission diffraction grating (Fig. 1 (a)): the periodicity of the structure leads to a splitting of the incident beam into several replica corresponding to the orders of diffraction.

The well-known free-space evolution of a beam can find analogs in the temporal domain. Indeed, the parabolic spectral phase induced by the dispersion of an ultrashort optical pulse is equivalent to the paraxial diffraction affecting the spatial propagation of a light beam [3-5]. This space/time duality has already been extremely fruitful and has stimulated numerous new concepts or interpretation in ultrafast optics such as temporal or spectral lenses [6-8], Fresnel lens [9], temporal telescope [10] or microscope [11]. Other experiments have established links between the Talbot carpet observed in diffraction and advanced applications for repetition-rate multiplication of ultrafast sources [12, 13]. However, no explicit study of the space / time duality for a simple periodic grating field has been reported so far. This is the scope of the present paper to fill this gap by providing a series of experimental results highlighting what we call a temporal dispersion grating.

\section{Grating space/time duality}

In the context of the scalar theory of diffraction (Fresnel's formalism) the figure of paraxial diffraction $I_{S}$ observed at a distance $L$ for a 1dimensional object is

$$
I_{S}(x, L)=\left|F^{-1}\left\{\mathcal{F}\left(U_{i n i}(x) t_{S}(x)\right) e^{-i \frac{\lambda L}{4 \pi} k_{x}^{2}}\right\}\right|^{2},
$$

where $x$ is the transverse coordinate, $\lambda$ the wavelength of light and $k_{x}$ the spatial frequency coordinate. $t_{s}(x)$ is the complex transmission function that affects $U_{\text {ini }}$ the input wave spatial profile. We consider here $t_{s}(x)$ as periodic with a period $\Lambda$ and a finite spatial extend $\Delta x$. $F$ and $F^{-1}$ denote the direct and inverse Fourier transform operations, respectively. The time analog of Eq.1 gives the evolution of the temporal intensity profile $I_{T}$ of an initial ultrashort pulse $U_{\text {ini }}(\tau)$, modulated by $t_{T}(\tau)$, after it has propagated over distance $L$ in a dispersive medium.

$$
I_{T}(\tau, L)=\left|F^{-1}\left\{\mathcal{F}\left(U_{i n i}(\tau) t_{T}(\tau)\right) e^{i \frac{\beta_{2}}{2} L \omega^{2}}\right\}\right|^{2},
$$

where $\beta_{2}$ stands for the second order dispersion coefficient; $\omega$ is the angular frequency and $\tau$ the temporal coordinate. In analogy with the spatial case, we consider $t_{T}(\tau)$ as being a periodic function of frequency $f_{\text {mod }}$ applied over a temporal window of duration $\Delta \tau$. We consider here modulations of the type $t s, T(\xi)=T(\xi) \exp (i \theta(\xi))$, with $T(\xi)$ the modulus, $\theta(\xi)$ the phase and $\xi$ standing for the space $(x)$ or time $(\tau)$ coordinate. The modulation can be amplitude only (sinusoidal $T(\xi)$ and $\theta(\xi)=0$ ) (Fig. 1 (b1)), phase only $(T(\xi)=1$ and sinusoidal $\theta(\xi)$ ) (Fig. 1 (b2)) or a combination of both (sinusoidal $T(\xi)$ and binary phase modulation 0 or $\pi)$ (Fig. 1 (b3)). For the sake of simplicity, we consider as input condition $U_{\text {ini }}(\xi)=1$, i.e. a plane wave (space case) or a continuous wave (time case), but the conclusions can be readily extended to any unchirped input waveform as long as its duration is significantly higher than $\Delta \tau$. 
(a)

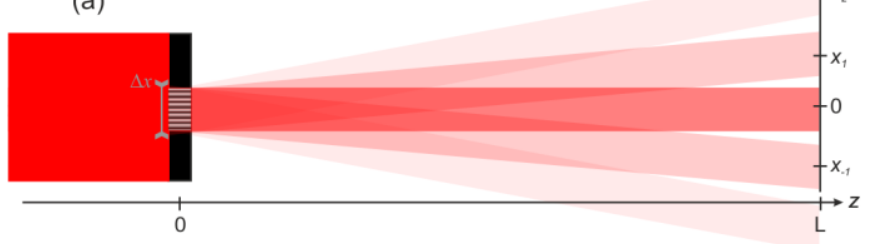

(b1)

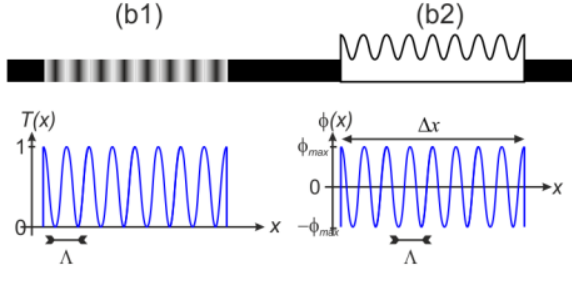

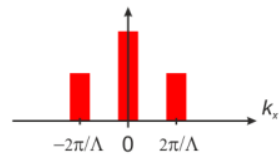

(c1)

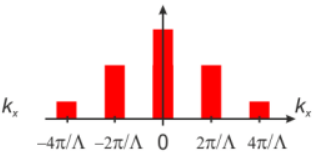

(c2) (b3)
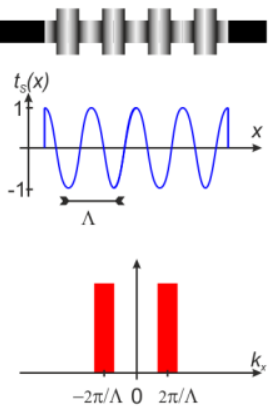

(c3)
Figure 1: (a) Spatial diffraction through a 1D transmission element. Transmissions considered in this work: sinusoidal amplitude only (b1), sinusoidal phase only (b2) and sinusoidal amplitude and binary [0, $\pi$ ] phase (b3) and their associated spectral domain representations (c).

Similar to the space grating that generated replica of the input field $U_{i n i}$ in the k space (Fig. 1 (a)), the temporal grating generates replica of $U_{\text {ini }}$ in the time domain. In order to clearly observe these temporal replicas one should operate in the far field conditions $\left(L>>L_{D}=\Delta t^{2} /\left|\beta_{2}\right|\right)$, i.e. accumulate a significant amount of dispersion. In this context of Fraunhofer diffraction, the temporal intensity profile becomes a scaled replica of the optical spectrum, which forms the basis of frequency-totime mapping schemes now widely used in ultrafast detection [14,15]. As the periodic modulation of $t_{T}$ leads to spectral sidebands equally spaced by $f_{\text {mod }}$, the longitudinal evolution of the position $\tau_{m}$ of the temporal sidebands are also equally spaced and are given by the simple analytical formula

$$
\tau_{m}=2 \pi m f_{\text {mod }} \beta_{2} L
$$

which is the temporal analog of the well-known law for gratings operating at normal incidence and small angles: $x_{m}=m L \lambda / \Lambda$. If the modulation period $1 / f_{\text {mod }}$ is short compared to $\Delta \tau$, the spectral width corresponding to the modulation envelope will be narrow compared to $f_{\text {mod }}$ and the temporal broadening of the envelope for the temporal replica will be moderate. Consequently, we can consider that the various temporal sidebands are separated if $\tau_{1}>\Delta \tau$. This condition can be fulfilled either by using a large enough accumulated dispersion $\beta_{2} L$, a high modulation frequency $f_{\text {mod }}$ or by limiting the temporal modulation extend $\Delta \tau$.

\section{Experimental implementation}

Figure 2 shows the setup built to observe temporal dispersion gratings. It relies on commercially-available components used in telecommunication. A continuous wave (CW) laser at $1550 \mathrm{~nm}$ is first modulated by a phase or intensity modulator driven by an electrical clock delivering a sinusoidal signal. The resulting temporal grating has a frequency $f_{\text {mod }}$ that we can vary between 12 and $40 \mathrm{GHz}$. In order to limit the temporal extent of the signal, the modulated wave passes through a second intensity modulator (Lithium Niobate) driven by an on-off keying pattern containing one ' 1 ' bit followed by thirty one ' 0 ' bits. An electrical frequency divider is used to obtain a temporal duration $\Delta \tau$ that is equal to four times the period of the frequency modulation (duration corresponding to $1 \mathrm{bit}$ ). Indeed, for our range of $f_{\text {mod }}$ explored and the level of dispersion $\beta_{2} L$ we can induce, we have found that $\Delta \tau=4 / f_{\text {mod }}$ is a practical trade-off between temporal replica separation and envelope broadening $[16,17]$.

Dispersion is achieved through the propagation in three spools of dispersion compensating fiber (DCF). The first fiber has a length of $11.5 \mathrm{~km}$ and a dispersion of $-100 \mathrm{ps} / \mathrm{km} / \mathrm{nm}$ whereas the length of the second and third segments are 13.5 and $6.5 \mathrm{~km}$ with dispersion of -124 and $-107 \mathrm{ps} / \mathrm{km} / \mathrm{nm}$, respectively. The integrated dispersion is $-3520 \mathrm{ps} / \mathrm{nm}\left(\beta_{2} L=4485 \mathrm{ps}^{2}\right)$. The DCFs could also be replaced by other dispersive elements able to provide a high level of dispersion such as linearly chirped fiber Bragg gratings [18]. In order to compensate for the optical losses, an erbium doped fiber amplifier (EDFA) was inserted between the first and second spools. The EDFA was combined with an optical bandpass filter (OBPF) to limit the accumulation of amplified spontaneous emission and the optical powers used were kept low enough (below $10 \mathrm{~mW}$ ) to avoid any non-linear effect such as self-phase modulation. An alternative to the use of a lumped EDFA is to take advantage of potential distributed Raman amplification [14]. The signal is analyzed in the temporal domain thanks to a high speed photodiode (electrical bandwidth of $70 \mathrm{GHz}$ ) combined with a sampling oscilloscope having a bandwidth of $70 \mathrm{GHz}$. The input and output spectrums are also recorded with a high resolution optical spectrum analyzer.

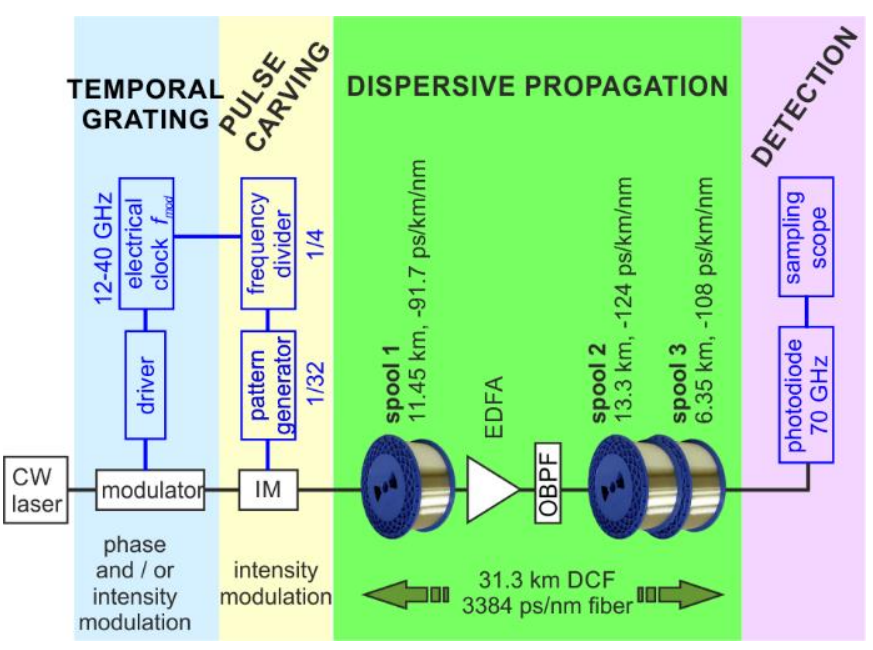

Figure 2: Experimental setup implemented to observe temporal dispersion gratings. CW: Continuous wave, IM: Intensity modulator, DCF: Dispersion compensating fiber, EDFA: Erbium-doped fiber amplifier, OBPF: Optical bandpass filter, OSA: Optical spectrum analyzer. 


\section{Experimental results}

\section{A. Amplitude only temporal dispersion grating}

Let us first discuss the results obtained with a modulation of the temporal intensity profile. The input and output experimental temporal profiles achieved for $f_{\text {mod }}=24 \mathrm{GHz}$ are plotted on Fig. 3 (a1) and Fig. 3 (a2), respectively. We clearly observe that the initial modulated burst splits into two temporal sidebands (Fig. 3 (a2)). The temporal profile is fully symmetric with respect to the central pulse, indicating that the effects of higher-order dispersion terms can be neglected. The experimental records are in qualitative agreement with numerical simulations Fig 3 (b1) (input modulated pulse) and Fig. 3 (b2) (far field). However, we can note some fast oscillations in the experiments recorded (Fig 3(a2)) that are not reproduced theoretically. Those oscillations are attributed to interferences with some residual CW background. Indeed, we checked in Fig. 3 (b3) that if we add in the simulation a residual continuous wave contribution as low as $0.25 \%$ of the input pulse peak power (corresponding to the extinction ratio of our second modulator), these fast oscillations are well reproduced. To remove the influence of these oscillations, we numerically applied a lowpass spectral filtering and obtained the signal shown in Fig. 3 (a3) in excellent agreement (both in terms of sideband location and level) with the theoretical signal (Fig. 3 (b2)).

Fig. 3(c) shows the evolution of the far field temporal intensity with $f_{\text {mod. }}$. The locations of the first sidebands evolve following a linear trend in quantitative agreement with the simple analytical law provided by Eq. 3 with $m= \pm 1$. Let us note that as increasing $f_{\text {mod }}$ leads in our setup to narrowing the duration of the central initial burst, it leads also to an increase of the central pulse obtained after the dispersive propagation.

\section{B. Phase only temporal dispersion grating}

We explore now the case of a temporal phase grating by using a sinusoidal phase modulation $\theta(\tau)=A \sin \left(2 \pi f_{\text {mod }} \tau\right)$ with $A=1.34 \mathrm{rad}$. Results recorded at the output of each DCF spool are displayed in Fig. 4(a) where we observe the progressive splitting of the initial superGaussian intensity profile into different temporal structures that are symmetrically located around the central peak. After DCF 1, the integrated dispersion is not enough to fully separate the various substructures. After DCF 2, we clearly observe two pairs of well-separated sidebands corresponding to the first and second temporal diffraction orders. The separation between those sidebands further increase during the propagation in the last spool. The temporal location of the sidebands are consistent which the prediction drawn from Eq. 3 with $m= \pm 1$ and \pm 2 . Compared to the previous case, the level of the first-order sideband has been considerably increased and the second order is now visible as being one tenth of the central pulse intensity. A third set of sidebands should in principle exist, but its theoretical level is below -20 $\mathrm{dB}$ and therefore not visible. The very different shape of the temporal output signal as compared to the amplitude only modulation comes from the spectra of the involved signals (see Fig. 1(c)). In the amplitude only modulation, the optical spectrum is essentially a spectral line at the carrier frequency surrounded by two low-amplitude lines spaced by $f_{\text {mod, }}$ with an amplitude reduced by $6 \mathrm{~dB}$ compared to the carrier. On the contrary, the optical spectrum associated to the phase only modulation is a series of spectral lines with an amplitude given by a $m$-th order Bessel function $J_{m}$ of the first kind $\left|J_{m}(A)\right|^{2}$ [19]. Therefore, as the dispersion leads in the far field to a frequency-to-time conversion, the differences in the spectra becomes readily apparent in the time domain and the recorded experimental levels of the sidebands are in excellent agreement with the analytical predictions. Note that adjusting the level of $A$ may enable to increase the number of visible sidebands and to generate at will temporal sequences containing triplets, quintuplets or more equally spaced pulses.
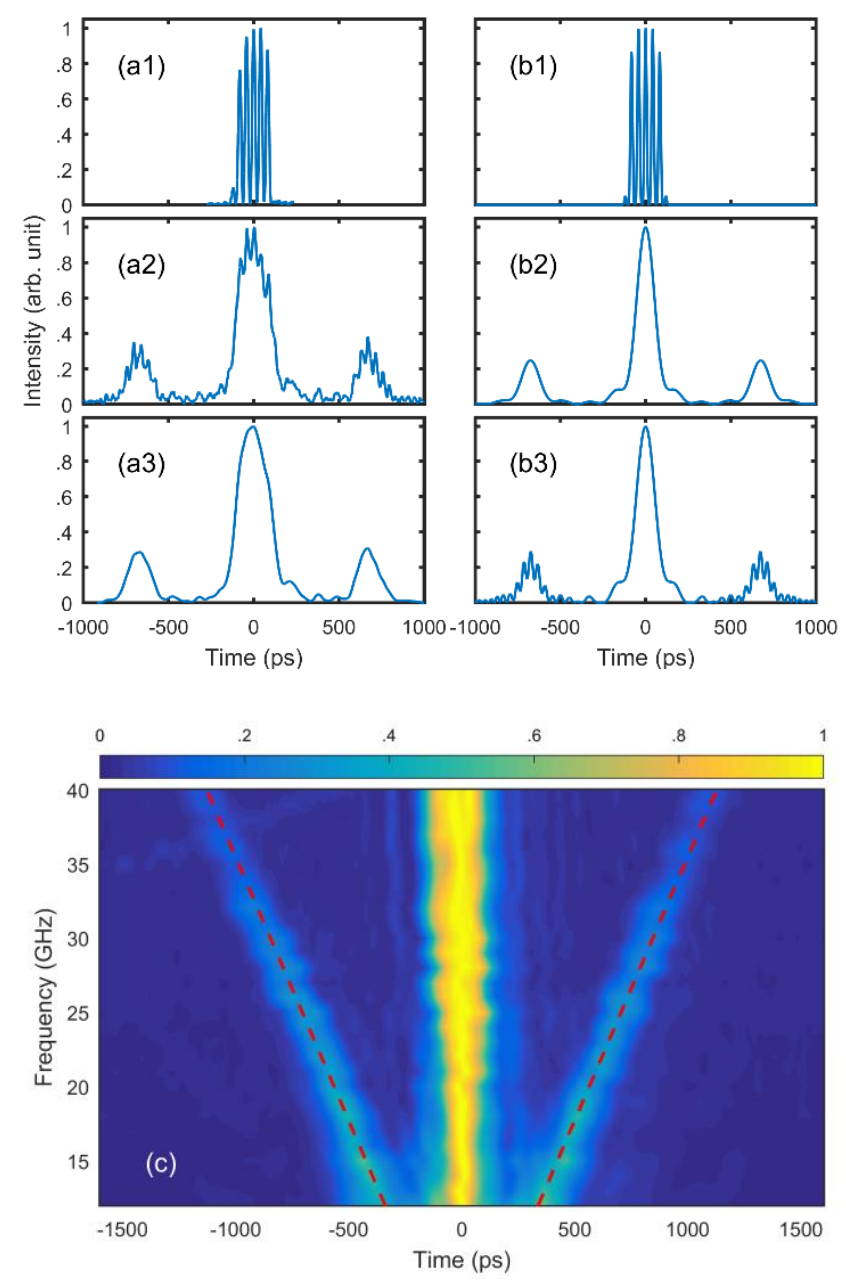

Figure 3: Amplitude only temporal dispersion grating. (a-b) Temporal intensity profiles at the input and output of the fiber segments (panels 1 and 2). Panels a are the experimental results whereas numerical simulations are plotted in panels $\mathrm{b}$. The numerical simulation taking into account a $0.25 \%$ residual CW background is plotted on (b3) and the experimental results after applying a lowpass spectral filtering is plotted in (a3). (c) Evolution of the output temporal intensity profile with the modulation frequency $f_{\text {mod. }}$. The theoretical predictions for the location of the $1^{\text {st }}$ order sidebands (Eq. 3) are plotted with a red dashed solid line.

Fig. 4 (b) shows the evolution of the far field temporal intensity with $f_{\text {mod. }}$ and confirms the quantitative agreement with the predictions given Eq. 3 for the sideband location. We can however note that, similarly to Fig. 3(c), the amplitude of the sidebands decrease for $f_{\text {mod }}>30 \mathrm{GHz}$. This is due to the bandwidth limitations of our phase modulator and RF driver that reduces the amplitude of the phase modulation. 


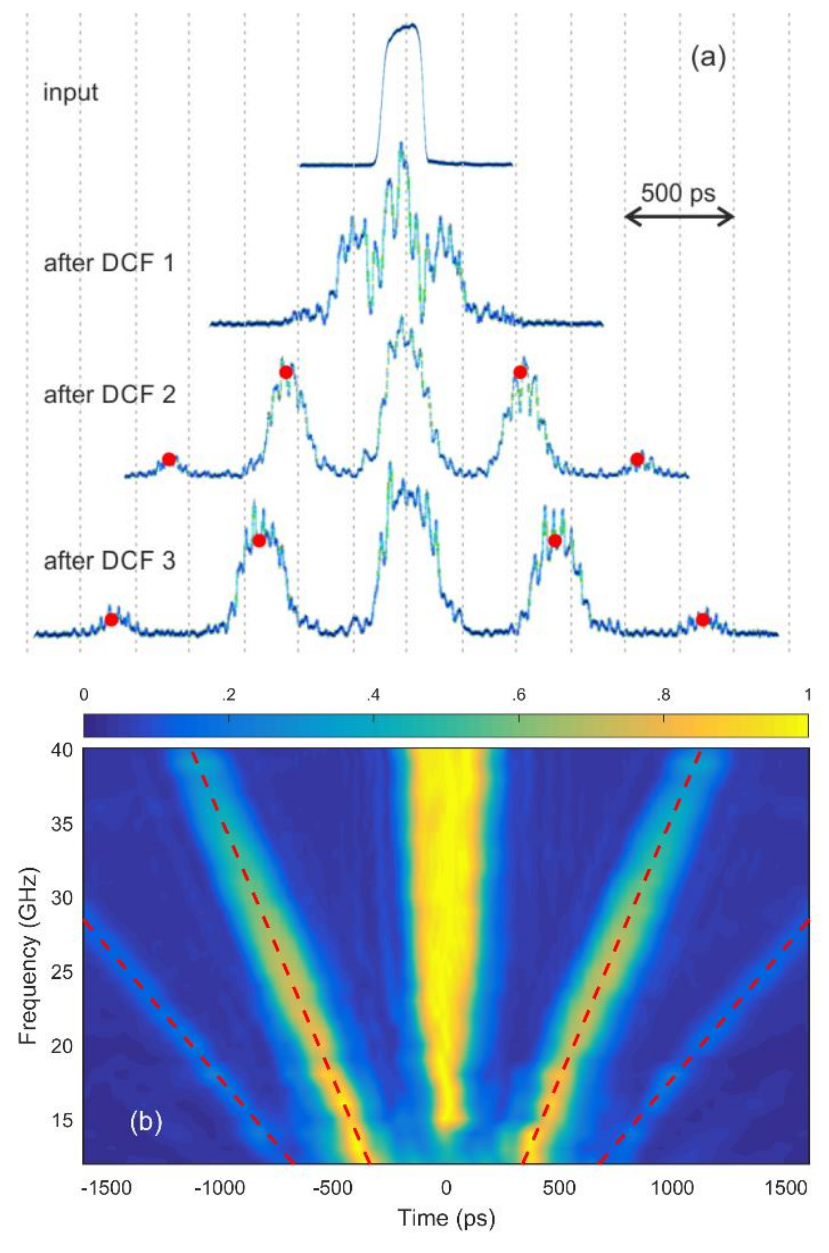

Figure 4: Phase only temporal dispersion grating. (a) Temporal intensity profiles taken at different positions. From top to bottom: at the input, after DCF 1, after DCF 2 and after DCF 3. The experimental records (screen copies of the oscilloscope) obtained for $f_{\text {mod }}=24 \mathrm{GHz}$ are compared with the theoretical prediction of the $1^{\text {st }}$ and $2^{\text {nd }}$ order sidebands (red circles, temporal location given by Eq. (3) and level given by $\left.\operatorname{Vm}(A)\right|^{2}$. (b) Evolution of the output temporal intensity profile with the modulation frequency $f_{\text {mod. }}$. The theoretical prediction of the location of the $1^{\text {st }}$ and $2^{\text {nd }}$ order sidebands (Eq. 3) are plotted with a red dashed solid line.

\section{Amplitude and phase temporal dispersion grating}

We finally investigated the evolution of a wave passing through a temporal grating combining both intensity and phase modulation. Indeed, by using an intensity modulator operating at the point of minimum transmission and driven by a frequency $f_{\text {mod, }}$ it is possible to obtain a modulation of the intensity profile at the frequency $2 f_{\text {mod }}$ (see inset of Fig. 5a) with a $\pi$ phase shift between two successive oscillations. The resulting wave is therefore carrier suppressed so that its central spectral component is canceled (see Fig. 1(c3) as well as the experimental record of Fig. 5(b), blue line). Let us mention that other methods to generate such carrier-suppressed spectra exist such as the use of a temporal triangular phase modulation [20] or a sinusoidal phase modulation with an amplitude of modulation $A=2.40 \mathrm{rad}$ (but in this case, the resulting spectrum will contain several sets of spectral bands). As a consequence, the temporal pattern that will be induced through dispersion will not contain any high-levelcentral peak and reduces to two symmetric sidebands associated to the first order dispersion. This feature is experimentally confirmed in the temporal output shape shown in Fig. 5(b) (orange curve). We compare this temporal measurement with the optical spectrum (blue curve, input and output spectra are identical due to the absence of nonlinear impairments): the temporal record stretched by $1 / \beta_{2} L$ is in agreement with the optical spectrum measured independently, which is consistent with the dispersive Fourier transform technics $[14,18]$.
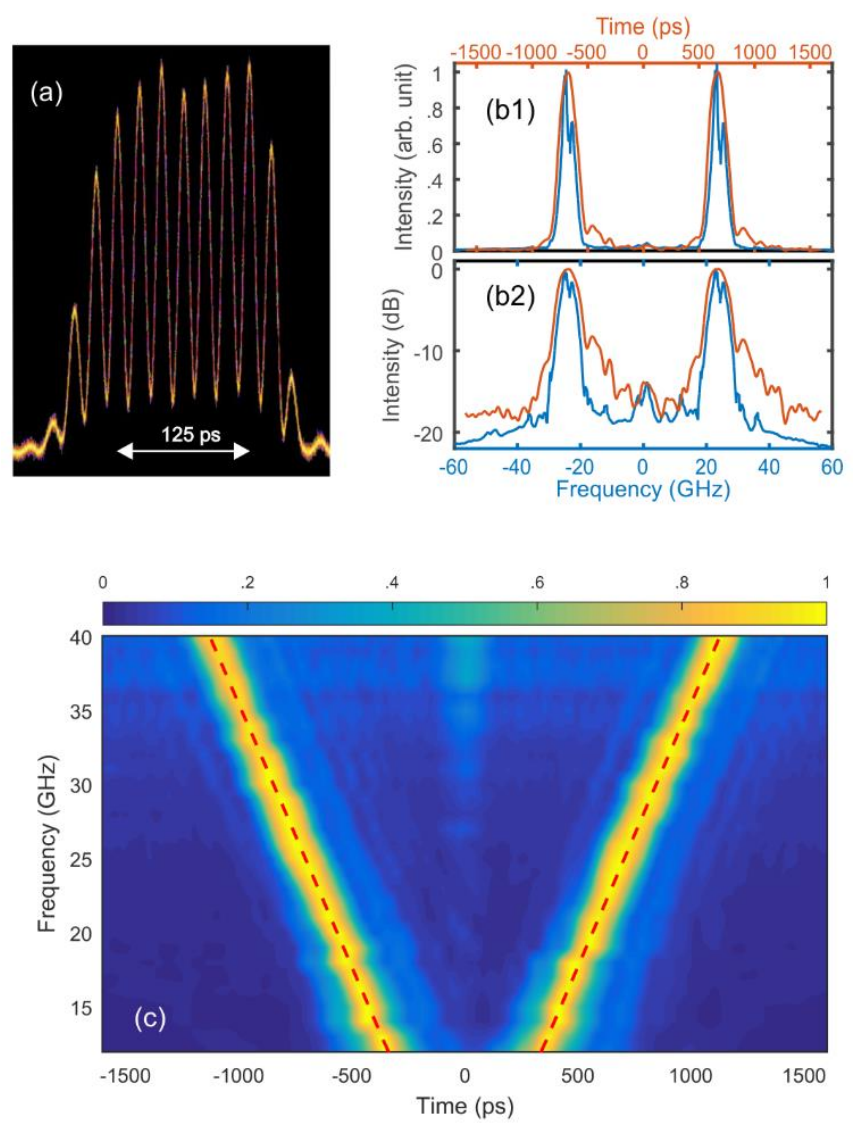

Figure 5: Amplitude and phase temporal dispersion grating. (a) Temporal intensity profile recorded at the input of DCF 1 on the high speed sampling oscilloscope for $f_{\text {mod }}=24 \mathrm{GHz}$.

(b) Temporal intensity profiles recorded at the output of DCF 3 (orange curves and orange horizontal axis). Results are plotted on a linear and logarithmic vertical scale (panels 1 and 2 respectively). They are compared with the optical spectrum of the signal directly recorded on the OSA $(1 \mathrm{GHz}$ resolution, blue curves and horizontal axis ) that is stretched by a factor $\beta_{2} L$. (c) Evolution of the output temporal intensity profile with the modulation frequency $f_{\text {mod. }}$. The theoretical prediction of the location of the $1^{\text {st }}$ order sidebands (Eq. 3) are plotted with a red dashed solid line.

The summary of the results obtained for various $f_{\bmod }$ provided in Fig. 5(c) confirms the generation after dispersive propagation of only two pulses with a spacing being directly proportional to $f_{\text {mod }}$ as anticipated from Eq. 3. Once again, we can observe a slight degradation of the pattern of $f_{\text {mod }}$ above $30 \mathrm{GHz}$ due to optoelectronics bandwidth limitation. 


\section{Conclusion}

To conclude, we have provided a clear experimental evidence of the link that can be drawn between the diffraction induced in the far field by a periodic spatial 1D structure and the temporal pattern resulting from the dispersion of a periodically modulated wave. We have tested three different temporal modulations and stressed how they affect the resulting temporal pattern. Using a phase and intensity modulation provided by a modulator working at the point of null transmission enables to cancel the energy contained in the zero $^{\text {th }}$-order temporal peak. Using other modulations schemes such as saw-tooth phase profiles induced through cross-phase modulation [21] one could improve the side lobes intensity and reproduce in the temporal domain the analog of blazed gratings. At a more fundamental level this work exemplifies once again the striking space-time duality existing between spatial diffraction and temporal dispersion.

Funding Information. Conseil Régional de Bourgogne-Franche-Comté (PARI Photcom)

Acknowledgment. We thank Julien Fatome for fruitful discussions and experimental assistance. This work has benefited from the PICASSO platform.

\section{References}

[1] M. Born, E. Wolf, Principles of Optics, Seventh Edition ed., Cambridge University Press1999.

[2] E. Hecht, Optics, 4th, International edition, Addison-Wesley, San Francisco, (2002).

[3] P. Tournois, J. Vernet, G. Bienvenu, Sur l'analogie optique de certains montages électroniques: Formation d'images temporelles de signaux électriques, CR Acad. Sci, 267 (1968) 375-378.

[4] J. van Howe, C. Xu, Ultrafast optical signal processing based upon space-time dualities, J. Lightw. Technol., 24 (2006) 2649-2662.

[5] B.H. Kolner, Space-time duality and the theory of temporal imaging, IEEE J. Quantum Electron., 30 (1994) 1951-1963.

[6] B.H. Kolner, M. Nazarathy, Temporal imaging with a time lens, Opt. Lett., 14 (1989) 630-632.

[7] E.R. Andresen, C. Finot, D. Oron, H. Rigneault, Spectral Analog of the Gouy Phase Shift, Phys. Rev. Lett., 110 (2013) 143902.

[8] L.K. Mouradian, F. Louradour, V. Messager, A. Barthélémy, C. Froehly, Spectrotemporal imaging of femtosecond events, IEEE J. Quantum Electron., 36 (2000) 795 801.

[9] L. Chantada, C.R. Fernández-Pousa, M.T. Flores-Arias, C. Gómez-Reino, Focusing of a train of pulses in phase opposition through a linear dispersive medium, J. Opt. A., 7 (2005) 767.

[10] M.A. Foster, R. Salem, Y. Okawachi, A.C. Turner-Foster, M. Lipson, A.L. Gaeta, Ultrafast waveform compression using a time-domain telescope, Nat. Photonics, 3 (2009) 581-585.

[11] P. Suret, R. El Koussaifi, A. Tikan, C. Evain, S. Randoux, C. Szwaj, S. Bielawski, Single-shot observation of optical rogue waves in integrable turbulence using time microscopy, Nat Commun, (2016) 13136.

[12] T. Jannson, J. Jannson, Temporal self-imaging effect in single-mode fibers, J. Opt. Soc. Am., 71 (1981) 1373-1376.

[13] J. Azaña, L.R. Chen, General temporal self-imaging phenomena, J. Opt. Soc. Am. B, 20 (2003) 1447-1458.

[14] D.R. Solli, J. Chou, B. Jalali, Amplified wavelength-time transformation for realtime spectroscopy, Nat. Photon., 2 (2008) 48-51.

[15] T. Jannson, Real-time Fourier transformation in dispersive optical fibers, Opt. Lett., 8 (1983) 232-234.

[16] J. Azaña, Temporal self-imaging effects for periodic optical pulse sequences of finite duration, J. Opt. Soc. Am. B, 20 (2003) 83-90.

[17] V. Torres-Company, D.E. Leaird, A.M. Weiner, Dispersion requirements in coherent frequency-to-time mapping, Opt. Express, 19 (2011) 24718-24729.
[18] M.A. Muriel, J. Azaña, A. Carballar, Real-time Fourier transformer based on fiber gratings, Opt. Lett., 24 (1999) 1-3.

[19] J.W. Goodman, Introduction to Fourier Optics, Roberts \& Co2005.

[20] N. Verscheure, C. Finot, Pulse doubling and wavelength conversion through triangular nonlinear pulse reshaping, Electron. Lett., 47 (2011) 1194-1196.

[21] F. Parmigiani, M. Ibsen, P. Petropoulos, D.J. Richardson, Efficient all-optical wavelength conversion scheme based on a saw-tooth pulse shaper, IEEE Photon. Technol. Lett., 21 (2009) 1837-1839. 